\title{
An exploration of the ballistic resistance of multilayer graphene polymer composites
}

\author{
M.R. O’Masta*, B.P. Russell and V.S. Deshpande \\ Engineering Department, University of Cambridge, Trumpington Street, Cambridge CB2 1PZ, UK
}

\begin{abstract}
The response of multilayer graphene / polyvinyl alcohol (MLG/PVA) films were studied under quasi-static (Q.S.) and dynamic, edge-clamped, transverse loading. The $10 \mu \mathrm{m}$ thick films, reinforced by $\sim 35$ vol.\% MLG and measuring $85 \mathrm{~mm}$ square, were fabricated by liquid exfoliation of the graphene followed by filtration of the MLG/PVA dispersion. The responses of the MLG/PVA films were compared with those of equal areal mass films of pure PVA and aluminum. The moderately conductive $\left(\sim 10^{-2} \mathrm{~S} \mathrm{~cm}^{-1}\right)$ MLG/PVA films had a Young's modulus approximately twice that of PVA and a low strain rate $\left(10^{-3} \mathrm{~s}^{-1}\right)$ peak strength that was about 50\% higher. Moreover, while the MLG/PVA films had a tensile strength lower than the Al films, they had a higher load carrying capacity compared to the Al films and were stiffer than the PVA films under Q.S. transverse loading. The ballistic limit of the MLG/PVA films was 50\% higher than the Al films, but the higher ductility of the parent PVA resulted in the pure PVA films having a higher ballistic resistance. The ballistic resistance of the MLG/PVA is well predicted by a membrane stretching analysis and this enables us to present an outlook on the ballistic resistance potential of graphene/PVA composites comprising aligned large flakes.

Keywords: Polymer-matrix composites; Graphene; Impact behavior; Mechanical properties.

* Corresponding author: M.R. O’Masta; E-mail address: mro27@cam.ac.uk
\end{abstract}




\section{Introduction}

Graphene is part of a larger class of 2D materials, which includes among others $\mathrm{BN}, \mathrm{MoS}_{2}$, $\mathrm{NbSe}_{2}$ and $\mathrm{TaS}_{2}$ [1]. These 2D materials, and graphene in particular, have received considerable recent attention due to their many exceptional properties, including $2.3 \%$ absorption in the white light spectrum, high surface area, very high thermal and electrical conductivity, impermeability to gas and exceptional mechanical stiffness and strength [2]. Potential applications of graphene range from flexible electronics and photonic devices to barrier films and paints to sensors and bio-applications [2]. Advanced multifunctional composites is an additional area that also is ripe to benefit from graphene's electrical and mechanical properties. This study assesses the response of high volume fraction graphene-polymer composites in edge-clamped quasi-static and dynamic loading, with an aim to reveal the potential of such composites for ballistic resistance applications.

The fabrication of composite systems requires large quantities of graphene at low production cost. This can be achieved using a top-down approach, based on the exfoliation of graphene from graphite, rather than the bottom-up approaches (e.g. chemical vapor deposition and epitaxial growth) that produce higher quality flakes [3]. Developed methods for mechanical exfoliation are sonication, ball milling and fluid dynamics [3]. Paton et al. [4] have shown the liquid exfoliation of graphene using high-shear forces can create large graphene quantities of consistent quality ( $10 \mathrm{~nm}$ thick multi-layer graphene platelets of $\sim 0.4 \mu \mathrm{m}$ in-plane dimensions). Furthermore, with the liquid exfoliation method, polymers can be introduced to stabilize the exfoliated graphene as part of the fabrication method.

Graphene has been used as a reinforcement in a range of thermosets and thermoplastics [57]. Very small volume fractions are needed to surpass the percolation limit and conductivity 
values above $0.01 \mathrm{~S} \mathrm{~cm}^{-1}$ are achievable from at most a few volume percent of graphene $[5,6]$. Furthermore, graphene reinforcement can increase the parent material's glass transition temperature, and cause large increases in the tensile strength and Young's modulus, albeit with significant decreases in ductility [6-8]. For instance, many studies using polyvinyl alcohol (PVA) as a filler (attractive for its solubility in water and ability to stabilize graphene during liquid exfoliation) have reported increases up to $100 \%$ in strength and modulus as well as moderate conductivity levels with the addition of a few volume percentage of graphene [9-13]. However, most studies have been limited to small strains and quasi-static loading.

The impact response of 10-100 nm thick sheets of pure multi-layer graphene (MLG) when impacted by a $\sim 4 \mu \mathrm{m}$ diameter silicon sphere was studied by Lee et al. [14]. The material behaved as a thin membrane and failed by a tensile "petalling" mode: such tensile governed failure modes are commonly observed in thin metal plates $[15,16]$ and fiber reinforced composite laminates with a low matrix shear strength [17-19]. Phoenix and Porwal [20] analytically solved the limiting impact velocity (commonly referred to as the ballistic limit $V_{b l}$ ) of such membrane type structures subject to normal impact. This predicted ballistic limit scales primarily with the tensile strength of the membrane. With graphene having an intrinsic strength of $130 \mathrm{GPa}$ [21] and a near isotropic in-plane response, a composite reinforced with graphene has the potential to surpass the composites of high performance fibers (e.g. aramids, UHMPWE, PBO and PIPD).

This study examines this potential by investigating the quasi-static (Q.S.) and dynamic transverse loading response of a $10 \mu \mathrm{m}$ thick multilayer graphene / polyvinyl alcohol (MLG/PVA) composite film with $\sim 35$ vol.\% MLG. We first report the tensile response and toughness of the composite. These measured material properties are used to interpret the measured transverse loading responses of the MLG/PVA films under Q.S. and dynamic 
(ballistic) loading, and these measurements are contrasted with the performances of equal areal mass films of pure PVA and $99 \%$ purity aluminum. Comparisons with an analytical membranestretching model are presented, and this model is then extended to present an outlook on the ultimate ballistic potential of graphene composites comprising large well-aligned flakes in PVA.

\section{Film materials and fabrication}

\subsection{Materials}

All films had an areal density of $a \approx 0.017 \mathrm{~kg} \mathrm{~m}^{-2}$ (see Table 1). The multilayer graphene / polyvinyl alcohol (MLG/PVA) composite film was fabricated from graphite powder (CAS: 7782-42-5; Fisher Scientific UK, Loughborough, UK) and PVA (MW: 27,000; CAS: 9002-895; Sigma Aldrich Company Ltd., Dorset, UK) using the procedure described subsequently. Fabrication of the pure PVA film followed the same procedure, with the exception of the graphite addition. The aluminum foil was purchased from Goodfellow Cambridge Ltd. (Huntingdon, UK).

Table 1: The areal density $a_{x}$, density $\rho_{x}$, and effective thickness $t_{x}$ of the three materials investigated here. The subscript ' $x$ ' denotes ' $c$ ', ' $p$ ' or ' $a l$ ' for the composite, PVA and Al films, respectively. The densities and conductivities of Al and PVA are quoted from Ref. [22].

\begin{tabular}{lccc}
\hline Material & $\begin{array}{c}a_{x} \\
\left(\mathrm{~g} \mathrm{~m}^{-2}\right)\end{array}$ & $\begin{array}{c}\rho_{x} \\
\left(\mathrm{~kg} \mathrm{~m}^{-3}\right)\end{array}$ & $\begin{array}{c}t_{x} \\
(\mu \mathrm{m})\end{array}$ \\
\hline MLG/PVA & $16.5 \pm 0.5$ & $1590 \pm 30$ & 10.3 \\
PVA & $17.4 \pm 0.1$ & 1190 & 14.6 \\
Al & $17.0 \pm 0.0$ & 2700 & 6.3 \\
\hline
\end{tabular}

\subsection{MLG/PVA fabrication}

Dispersion production: We liquid-exfoliated polymer-stabilized graphene following the procedure introduced in Paton et al. [4]. First, a $25 \mathrm{~g}$ mass of graphite powder was added to a 
$250 \mathrm{~mL}$ solution of $0.4 \mathrm{wt} . \%$ PVA in distilled water. Next, to exfoliate the graphite, the solution was mixed by a model L5M high-shear laboratory mixer (Silverson Machines Ltd., Chesham, UK) equipped with a $25.4 \mathrm{~mm}$ outer diameter, square hole high shear screen and operated at 7000 RPM for $90 \mathrm{~min}$. The dispersion was then centrifuged for $30 \mathrm{~min}$. at $1950 \mathrm{RCF}$ to encourage settling of the unexfoliated graphite. Finally, the supernatant was collected for filtration.

Filtering equipment: Recovery of a composite film from the dispersion was carried out using the pressure filtration system sketched in Fig. 1. An aluminum grate with a hexagonal pattern of $1 \mathrm{~mm}$ diameter holes with a $1.3 \mathrm{~mm}$ center-to-center spacing was placed at the bottom of the vessel. Two filter papers were placed on top of this grate: (i) a 2-3 $\mu \mathrm{m}$ pore size qualitative filter paper (grade 55; Fisher Scientific UK) in contact with the grate and (ii) a $0.2 \mu \mathrm{m}$ pore size, Whatman Nuclepore membrane (purchased from Sigma Aldrich Company Ltd.) on top of this filter paper. The dispersion was then poured onto these filter papers as shown in Fig. 1. The Nuclepore membrane resisted passage of the PVA and graphene platelets while the qualitative filter paper and aluminum grate provided flexural support. Rubber stoppers placed between the vessel body and the filter papers, around the borders, prevented fluid leakage. 


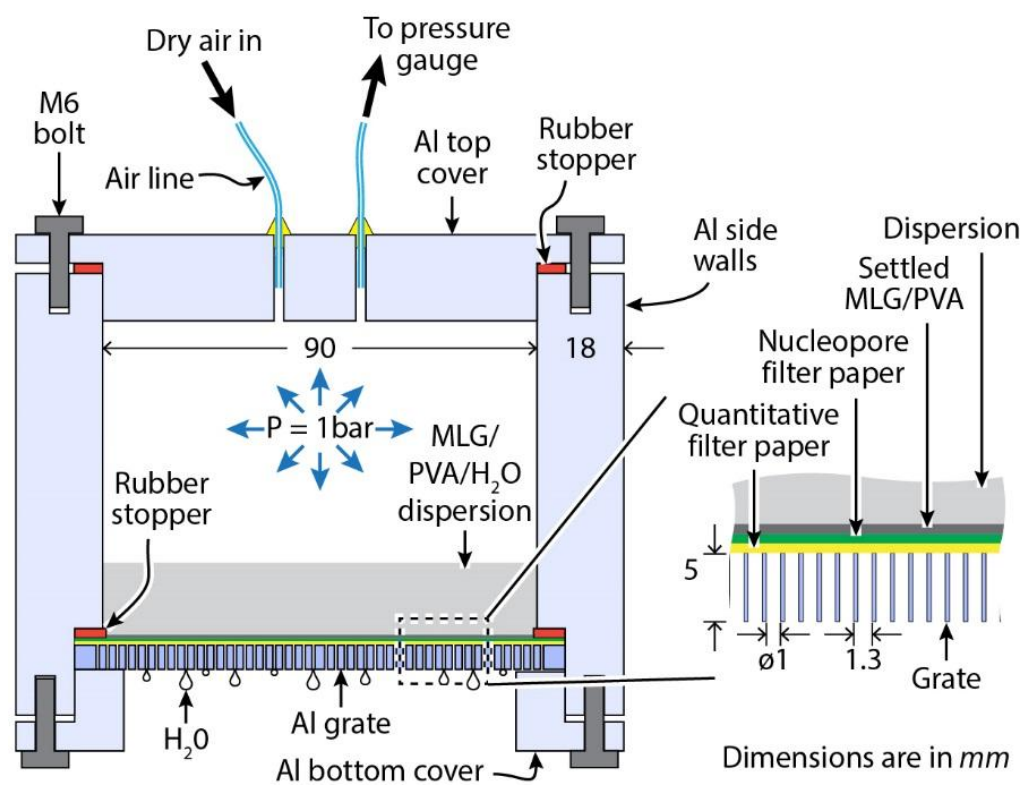

Figure 1: Sketch of the filtration equipment for fabrication of the MLG/PVA composite films.

Filtering procedure: A $250 \mathrm{~mL}$ volume of the dispersion was poured into the pressure vessel. The internal pressure was raised to 1 bar and held until the liquid filtered from the vessel ( $12 \mathrm{hr}$.). The Nuclepore membrane, with the hydrated MLG/PVA film coating, was removed from the vessel and placed under a lid to dry for $24 \mathrm{hr}$. The dried MLG/PVA film was then separated from Nuclepore membrane and stored in a dehumidifier.

\section{Physical and mechanical properties}

An image of the resulting MLG/PVA film is shown in Fig. 2a. The dispersion side of the film is dimpled from the filtering procedure (see Fig. 2b). A through-thickness cross-section of a sample torn due to impact loading is shown in Fig. 2c. There is distinct layering associated with the alignment of the MLG platelets in the plane of the film. 
(a) Macroscopic view

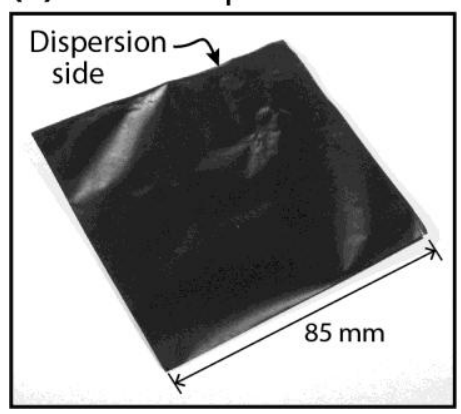

(b) Dispersion side

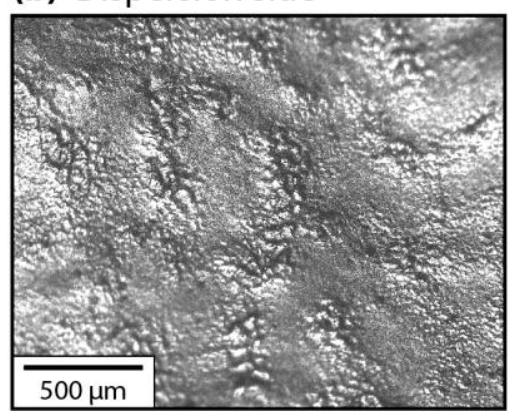

(c) Impact sample cross-section

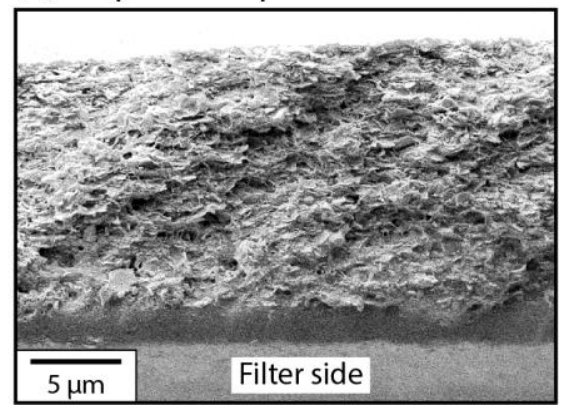

Figure 2: (a) Oblique photograph of a MLG/PVA film. (b) Optical image of the dispersion side of the film. (c) Through-thickness, cross-sectional SEM micrograph of a film torn by the impact of an $m_{p}=20 \mathrm{mg}$ projectile traveling at $V_{0}=16.3 \mathrm{~m} \mathrm{~s}^{-1}$.

The measured mass per unit area, or areal density $a_{c}$, of the film is listed in Table 1. Also shown is the measured density $\rho_{c}$ of a $100 \mathrm{mg}$ sample of the MLG/PVA film using helium pycnometry (performed by Quantachrome UK Ltd., Hartley Wintney, UK). These two measurements allow the calculation of the mean film thickness $t_{c} \equiv a_{c} / \rho_{c}$ as listed Table 1. Moreover, assuming a negligible void volume within the films, the volume fraction $\phi_{g}$ of the graphene within the films can be obtained from the rule of mixtures as

$$
\phi_{g}=\frac{\rho_{c}-\rho_{p}}{\rho_{g}-\rho_{p}}
$$

where $\rho_{c}=1590 \pm 30 \mathrm{~kg} \mathrm{~m}^{-3}, \rho_{g}=2260 \mathrm{~kg} \mathrm{~m}^{-3}$ and $\rho_{p}=1190 \mathrm{~kg} \mathrm{~m}^{-3}$ are the densities of the composite, graphene and PVA, respectively. This gives an upper bound estimation of $\phi_{g}=0.37 \pm 0.03$ (where the uncertainty is associated with the pycnometry measurement error of $\left.\rho_{c}\right)$.

The MLG/PVA film was also identified to be moderately conductive, with a conductivity of $\Sigma_{c} \sim 1-4 \mathrm{~S} \mathrm{~m}^{-1}$ (over five different samples using the experimental details in Appendix A). 
This conductivity value is similar to other graphene and graphene-oxide composites studied in the literature $[5,6]$ and is in contrast to the insulating nature of PVA as seen in Table 2.

Table 2: The mechanical and electrical properties of the three materials investigated here. The subscript ' $x$ ' denotes ' $c$ ', ' $p$ ' or ' $a l$ ' for the composite, PVA and Al films, respectively. The densities and conductivities of Al and PVA are quoted from Ref. [22].

\begin{tabular}{|c|c|c|c|c|c|c|}
\hline \multirow[b]{2}{*}{ Material } & \multicolumn{2}{|c|}{$\begin{array}{c}\text { Tension } \\
\left(\dot{\varepsilon}=10^{-3} \mathrm{~s}^{-1}\right)\end{array}$} & \multicolumn{2}{|c|}{$\begin{array}{c}\text { Tension } \\
\left(\dot{\varepsilon}=10^{-1} \mathrm{~s}^{-1}\right)\end{array}$} & \multirow{2}{*}{$\begin{array}{c}\text { Tear } \\
\text { resistance } \\
T_{x} \\
\left(\mathrm{~kJ} \mathrm{~m}^{-2}\right)\end{array}$} & \multirow{2}{*}{$\begin{array}{c}\text { Electrical } \\
\text { conductivity } \\
\sum_{x} \\
\left(\mathrm{~S} \mathrm{~m}^{-1}\right)\end{array}$} \\
\hline & $\begin{array}{c}E_{x} \\
(\mathrm{GPa})\end{array}$ & $\begin{array}{c}\sigma_{x} \\
(\mathrm{MPa})\end{array}$ & $\begin{array}{c}E_{x} \\
(\mathrm{GPa})\end{array}$ & $\begin{array}{c}\sigma_{x} \\
(\mathrm{MPa})\end{array}$ & & \\
\hline MLG/PVA & $6 \pm 0.6$ & $55 \pm 5$ & $8 \pm 0.7$ & $90 \pm 3$ & $2.9 \pm 0.1$ & $2 \pm 1$ \\
\hline PVA & $2.5 \pm 0.3$ & $35 \pm 4$ & $5 \pm 0.4$ & $120 \pm 5$ & $11.5 \pm 0.6$ & $1 \times 10^{-10}$ \\
\hline $\mathrm{Al}$ & $35 \pm 1$ & $182 \pm 5$ & $35 \pm 1$ & $185 \pm 3$ & $3.7 \pm 0.1$ & $3 \times 10^{7}$ \\
\hline
\end{tabular}

\subsection{MLG platelet thickness}

Procedure: X-ray diffraction was used to confirm the presence of MLG within the composite system and measure the MLG platelet thickness. Acquisition of the diffraction pattern employed a Philips X'Pert PW3020 X-ray diffractometer running a Bragg-Brentano scan in reflection mode. The X-ray radiation source was monochromated $\mathrm{Cu} \mathrm{K \alpha}$ (wavelength of $\lambda=$ $0.154 \mathrm{~nm}$ ), generated using an accelerating voltage of $40 \mathrm{kV}$ and a tube current of $40 \mathrm{~mA}$. Samples, measuring $30 \mathrm{~mm} \times 30 \mathrm{~mm}$, were mounted flat against a silicon wafer, and scanned over a diffraction angle of $2 \theta=14^{\circ}$ to $34^{\circ}$ in steps of $0.05^{\circ}$.

Results: Figure 3 shows a representative diffraction pattern of the MLG/PVA film. The broad diffraction peak at $2 \theta \approx 19^{\circ}$ was from PVA, as verified from a separate scan of the pure PVA film (Fig. 3). Using Bragg's law (i.e. $\lambda=2 d_{h k l} \sin \theta_{h k l}$ ), the peak at $2 \theta=26.4^{\circ}$ is identified as the (002) peak of graphite, with an interatomic spacing of $d_{002}=3.37 \AA$. For small crystals, a lower bound estimate of the crystal size normal to the diffraction plane can be calculated from the full-width half-mass, $B$, of the diffraction curve, using Scherrer's formula: 
$l_{h k l}=K \lambda /\left(B \cos \theta_{h k l}\right)$, where $K$ is a shape factor with a typical value of 0.9 [23]. Instrumental line broadening was $<0.1^{\circ}$, as measured using a LaB6 standard, and, as such, considered negligible (within experimental error). From an average of five samples, the interatomic spacing was $0.84^{\circ} \pm 0.07^{\circ}$, revealing of an average of $29 \pm 3$ graphene layers per graphite platelet (given by $l_{002} / d_{002}$ ). Thus confirming the presence of $\sim 30$ graphene layers graphene (MLG) platelets within the composite film, which is consistent with the AFM measurements from Ref. [4], whose liquid-exfoliation method for the graphene was replicated here.

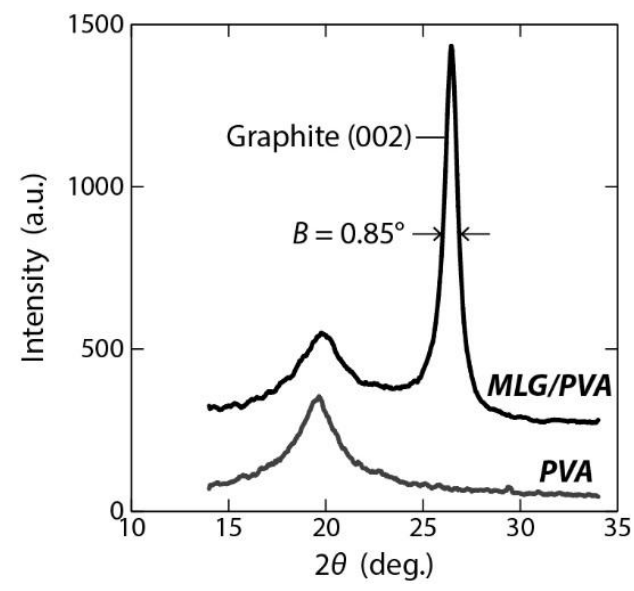

Figure 3: Representative X-ray diffraction patterns of MLG/PVA and PVA films. The graphite (002) full-width half mass, $B$, is identified.

\subsection{Tensile behavior}

Methodology: Rectangular samples $1.3 \mathrm{~mm}$ wide were cut by razor blade. Paper tabs were glued to either end to give a $20 \mathrm{~mm}$ gauge length between the tabs. The tabs were gripped using rubber lined friction grips attached to a model 5544 Instron (High Wycombe, UK), equipped with a $5 \mathrm{~N}$ load cell. Each sample was pulled at a constant rate of displacement corresponding to a nominal strain rate of either $\dot{\varepsilon}=10^{-3}$ or $10^{-1} \mathrm{~s}^{-1}$. Five repeat tests were conducted in each case. 
Results: The nominal stress versus strain responses of the MLG/PVA film are shown in Fig. 4a for two representative tests at each applied strain rate $\dot{\varepsilon}$. At $\dot{\varepsilon}=10^{-3} \mathrm{~s}^{-1}$, the material is approximately linear elastic up to a peak stress at $\varepsilon \sim 1.5 \%$. This is followed by a softening response with catastrophic failure at $\varepsilon \sim 4 \%$. The modulus $E_{c}$ and peak strength $\sigma_{c}$ values are tabulated in Table 2. At the high strain rate of $\dot{\varepsilon}=10^{-1} \mathrm{~s}^{-1}, E_{c}$ and $\sigma_{c}$ increase by $30 \%$ and $60 \%$, respectively (see Table 2), and the material response switches to elastic-brittle (see Fig. 4a).

(a) MLG/PVA film

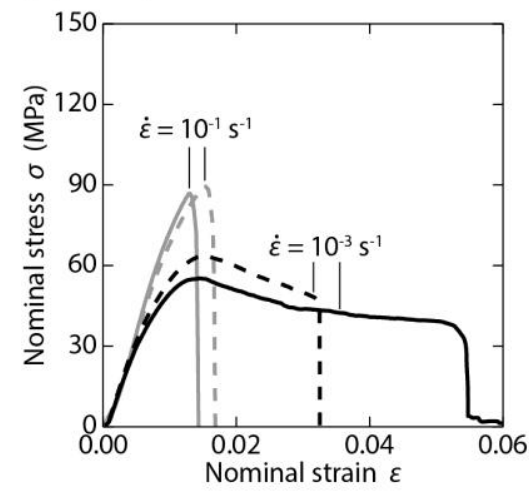

(b) PVA film

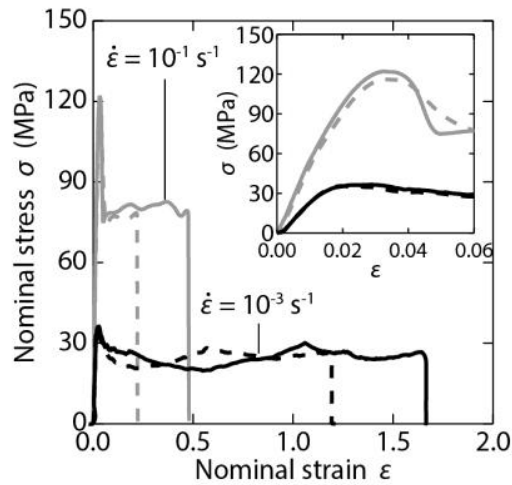

(c) Al film

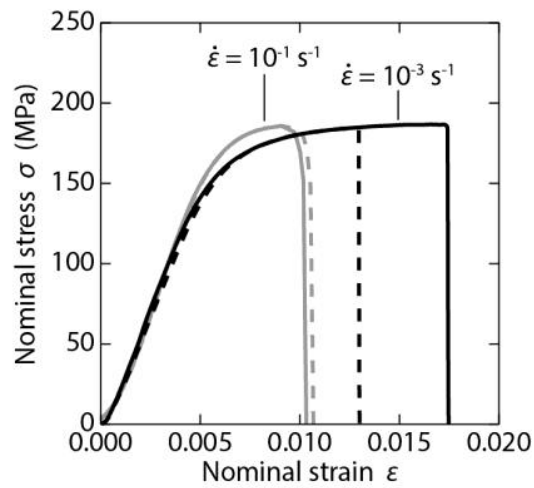

Figure 4: The uniaxial nominal tensile stress versus strain responses of the (a) MLG/PVA (b) PVA and (c) Al films. Two representative curves are shown for each applied strain rate $\dot{\varepsilon}$. In (b) an inset showing the early strain response is included.

The modulus and peak strength of the PVA film strained at $\dot{\varepsilon}=10^{-3} \mathrm{~s}^{-1}$ were substantially lower than the values measured for the MLG/PVA composite films (Fig. 4b). However, the PVA film was more ductile, with a ductility $>100 \%$. Further, the PVA films had a significantly higher strain rate sensitivity with its peak strength increasing by nearly a factor of four as the strain rate increased from $\dot{\varepsilon}=10^{-3}$ to $10^{-1} \mathrm{~s}^{-1}$. In fact at these high strain rates, the PVA film had a 33\% higher peak strength compared to the MLG/PVA film (Table 2). 
Conversely, the $\mathrm{Al}$ foil showed little if any strain rate sensitivity (Fig. 4c) but had a considerably higher modulus and peak strength compared to the MLG/PVA film and a very low ductility.

The ultimate graphene composite: "Ultimate" graphene/polymer composites are those comprised of defect-free, single layer graphene flakes of sizes in excess of the shear lag length (calculated to be on the order of $10 \mu \mathrm{m}$ [24]) that are perfectly aligned with the loading direction. Such composites will have an in-plane modulus $E_{c}$ and strength $\sigma_{c}$ given by the rule of mixtures:

$$
E_{c}=\phi_{g} E_{g}+\left(1-\phi_{g}\right) E_{p} \quad \& \quad \sigma_{c}=\phi_{g} \sigma_{g}+\left(1-\phi_{g}\right) \sigma_{p}
$$

where $E_{g}$ and $E_{p}$ are the moduli of the graphene flakes and polymer matrix, respectively, and $\sigma_{g}$ and $\sigma_{p}$ are their respective strengths. The basic material parameters are well established, i.e. $\sigma_{g}=100 \mathrm{GPa}$ and $E_{g}=1000 \mathrm{GPa}$ for defect-free graphene flakes [21] and $\sigma_{p}=100 \mathrm{MPa}$ and $E_{p}=5 \mathrm{GPa}$ for PVA. For the $\phi_{g}=0.35$ composite considered here, the predicted modulus and strength of the ultimate composite is $E_{c}=350 \mathrm{GPa}$ and $\sigma_{c}=35 \mathrm{GPa}$, respectively.

The properties of the graphene composites manufactured and tested are well below these upper bound predictions, with measured values of $E_{c}=6 \mathrm{GPa}$ and $\sigma_{c}=0.05 \mathrm{GPa}$. The primary causes for this poor performance are thought to be related to: (i) the small in-plane platelet sizes $(\sim 0.4 \mu \mathrm{m}[4])$ and the large number $(\sim 30)$ of graphene layers comprising a platelet mean the platelets are much smaller than the sizes required by shear-lag theory for efficient load transfer into the graphene; and (ii) relatively poor alignment of the platelets in the plane of the films. Additional effects such as the presence of defects, such as voids, are also expected to play a role in reducing the strength and modulus.

\subsection{Toughness}

Methodology: The tear resistance ("trouser tear" toughness) was performed using the trouser tear test as given by ASTM standard D1938 [25]. The coupon measured $12 \mathrm{~mm} \times 36 \mathrm{~mm}$ 
and had a $24 \mathrm{~mm}$ slit down the centerline of the long axis (Fig. 5). Each leg was gripped by a wedge action grip, and the coupon pulled apart in tension at a constant rate of displacement of $\dot{u}=250 \mathrm{~mm} \mathrm{~min}^{-1}$ by a model 5544 Instron equipped with a $5 \mathrm{~N}$ load cell. A representative response of each film is shown in Fig. 5. The steady-state load normalized by the film halfthickness gives the tear resistance.

(a) Schematic

(b) Response
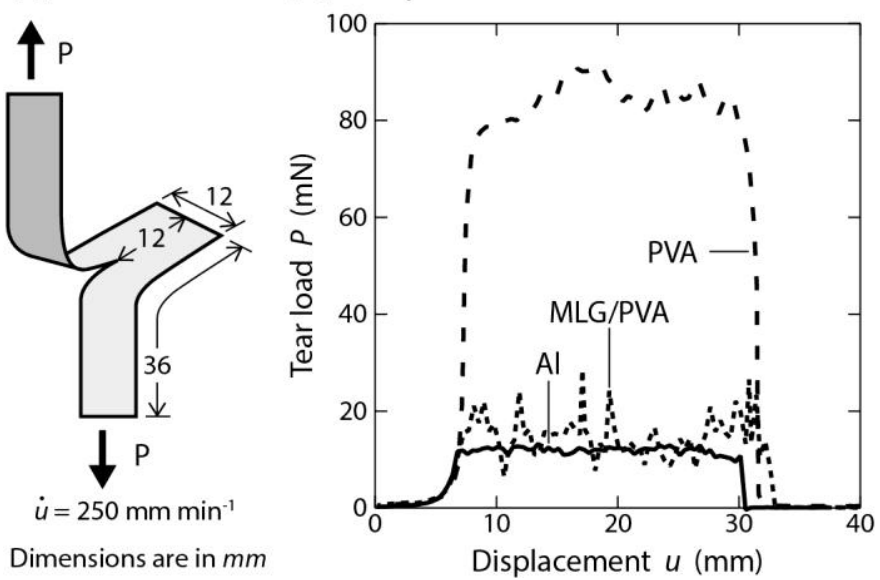

Figure 5: (a) Sketch of the trouser tear toughness experiment, and (b) representative load $P$ versus displacement $u$ responses of the three films investigated here.

Results: The tear toughness $T_{x}$ for each material $x$ reported in Table 2 is the ratio of the measured plateau load and the half thickness of the film averaged over three repeat tests. The PVA film was the toughest, consistent with the large ductility observed from the tensile measurements. The MLG/PVA and Al films had a similar toughness that was only $25 \%$ of the PVA value.

\section{Transverse loading test protocol}

The main aim of this study is to investigate the ballistic potential of MLG/PVA composites. However, given the observed strain rate sensitivity of the tensile response (Table 2), the transverse loading of the films was investigated under two conditions: (i) application of a 
constant low displacement rate, and (ii) projectile impact at a given (initial) impact velocity. In both cases, a film measuring $40 \mathrm{~mm} \times 40 \mathrm{~mm}$ was clamped between an aluminum plate containing a $30 \mathrm{~mm}$ diameter hole and a $0.5 \mathrm{~mm}$ thick steel ring with inner and outer diameters 30 and $60 \mathrm{~mm}$, respectively, on a pitch circle of diameter $45 \mathrm{~mm}$ (Fig. 6a). The clamping pressure was provided by twelve equally spaced circular magnets of diameter $8 \mathrm{~mm}$ embedded in flat bottom holes within the aluminum plate. The surface of the steel ring in contact with the film was lined with a $0.5 \mathrm{~mm}$ rubber sheet to increase the friction and evenly distribute the clamping pressure.

(a) Sample fixture

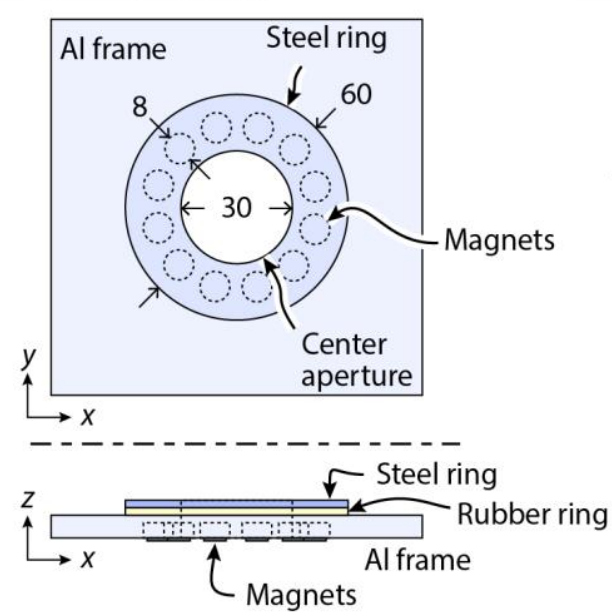

(c) Projectile impact test

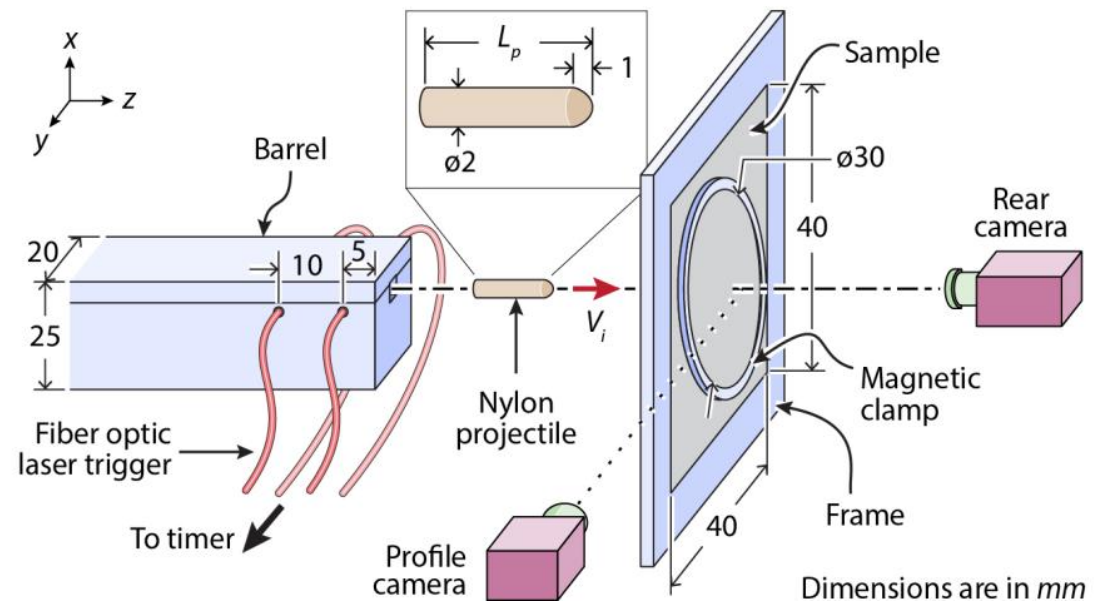


Figure 6: Sketches of (a) the clamping fixture, (b) the constant rate of displacement (CRD), Q.S. loading test and (c) a side view of the projectile impact test, showing the target and gas gun end.

\subsection{Quasi-static transverse loading}

The clamped films were loaded centrally and normally by a hemi-spherically tipped nylon rod of diameter $d_{p}=2 \mathrm{~mm}$. The indenter rod was attached to a 5544 model Instron and displaced at a rate $\dot{w}=1 \mathrm{~mm} \mathrm{~min}^{-1}$, and the applied load measured via the $5 \mathrm{~N}$ load cell of the test machine. Loading continued until a film was perforated.

\subsection{Projectile impact}

Cylindrical nylon rods of diameter $d_{p}=2 \mathrm{~mm}$ and length $L_{p}$, with a hemispherical tip, impacted the films centrally and normally (Fig. 6c) at a velocity $V_{0}$. The projectile was launched by a custom designed two-compartment gas-gun as sketched in Fig. 7. A type 6011 Burkert (Ingelfingen, Germany) solenoid valve released a reservoir of pressurized air that accelerated a sabot-encased rod (collectively referred to as a "ram") down the first compartment of the barrel. At the end of the first compartment, the ram impacted the projectile resting in the narrower second compartment and accelerated the projectile down the remainder of the barrel. This two compartment set-up prevented air from loading the film prior to impact by the projectile. Both the reservoir pressure (variable between 8 and 21 bar) and the ram position along the $250 \mathrm{~mm}$ long first compartment of the barrel controlled the impact velocity $V_{0}$ of the projectile.

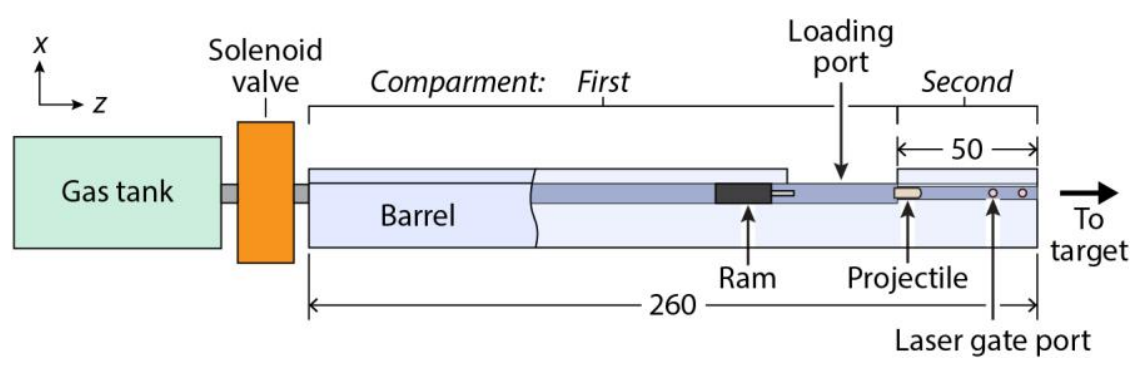


Figure 7: A side-view sketch of the gas-gun used for the projectile impact experiment. A cut away shows the interior of the double barrel. All dimensions are in $\mathrm{mm}$.

The target was placed $30 \mathrm{~mm}$ down range from the muzzle end of the barrel as shown in Fig. 6c. This was sufficiently close to preclude yaw of the projectile or a reduction in projectile velocity from air drag. A model v1610 Phantom (Vision Research, Bedford, UK) high-speed camera aligned normal to the film captured the deformation of the rear surface. A pair of laser gates separated by $10 \mathrm{~mm}$ (Fig. 6c and 7) was used to estimate the velocity of the projectile as it exited the barrel. A few select tests, with the high-speed camera set up for a side view, were performed to confirm that these exit velocity measurements were equal to the velocity $V_{0}$ at which the projectile impacted the films.

The length $L_{p}$ sets the mass $m_{p}$ of the projectile. The tests were conducted with $m_{p}=6$, 20 and $36 \mathrm{mg}$ (the $6 \mathrm{mg}$ projectile was a $2 \mathrm{~mm}$ diameter sphere). Following Cunniff [26], we present results in terms of the non-dimensional areal mass ratios of the film to the projectile, i.e. $\Gamma_{0}=\rho_{a} \pi d_{p}^{2} /\left(4 m_{p}\right)=\{8.9,2.7$ and 1.5$\} \times 10^{-3}$. Tests were conducted by increasing $V_{0}$ (in steps of $1.5 \mathrm{~m} \mathrm{~s}^{-1}$ ) for a given $\Gamma_{0}$ until the films failed, with failure defined as the appearance of a through-thickness crack in the post-impact inspection of the specimen. The mean of the highest $V_{0}$ where the film survived and the lowest $V_{0}$ where the film failed is defined as the ballistic limit $V_{b l}$.

\section{Quasi-static transverse loading}

The measured applied load $P$ versus indenter displacement $w$ response of the MLG/PVA film is plotted in Fig. 8a (for clarity only a single representative measurement from the three tests is included). The displacement $w \gg$ film thickness, implying that the response is dominated by the stretching of the film with negligible contribution from film bending. This results in an 
increasing slope $d P / d w$ as the film deflects and aligns with the applied load. A peak load is attained at $P \approx 0.5 \mathrm{~N}$ and the applied load then drops catastrophically to about $0.05 \mathrm{~N}$. This is associated with the formation of petals, i.e. radial cracks (Fig. 8b.i), and these cracks then propagate at an almost constant applied load [27] until complete failure of the film at $w \approx 2 \mathrm{~mm}$.

(a) Response

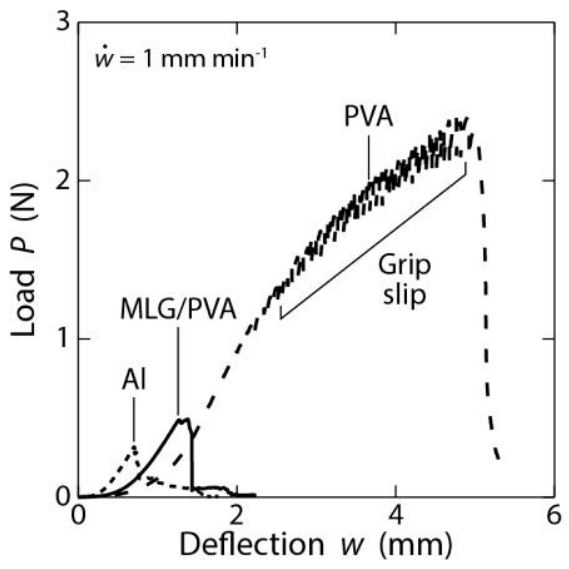

(b) Optical images of failed specimens

(i) $M L G / P V A$ film

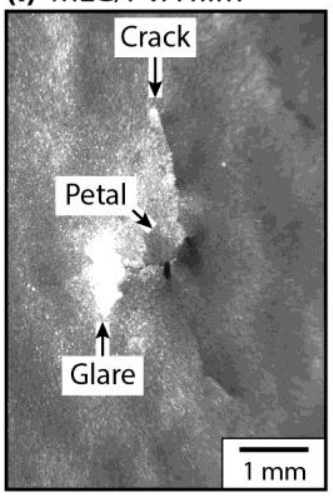

(ii) PVA film

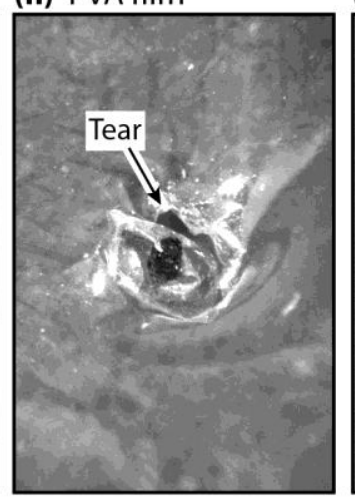

(iii) Al film

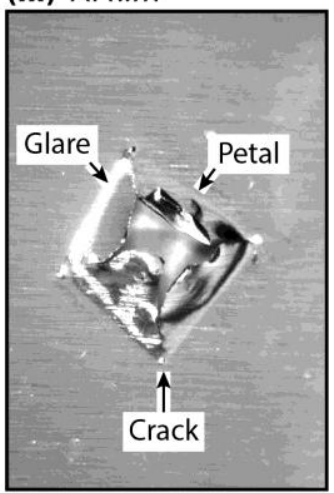

Figure 8: (a) Representative load-displacement responses of the three materials under quasi-static transverse loading. (b) Post-mortem optical images of the rear surfaces of the three sample types. Artifacts as a result of glare are marked along with the observed cracks.

The lower PVA modulus implied that the PVA film was initially weaker under transverse loading, but its higher ductility allowed the film to deflect more compared to the MLG/PVA film and better align with the load, thereby reaching a higher peak load. Moreover, the failure of the film at peak load was by the formation of a circumferential crack (Fig. 8b.ii) allowing the indenter to push through the film immediately on the formation of this crack. We note that the large forces sustained by the PVA film resulted in slippage of the film at grips giving rise to a saw-tooth portion of the $P-w$ curve as marked in Fig. 8a. This slippage allowed further alignment of the film with the load as more material was dragged from within the grips towards the applied load. Thus, we anticipate the displacement at the failure of the film (and 
consequently the applied load) is higher in these experiments compared to the case if grip slippage was inhibited.

The Al film had a response similar to the MLG/PVA film though the higher modulus of Al implied that it was initially stiffer as seen in Fig. 8a. However, the Al film had a lower failure load which was due to: (i) the higher modulus of the Al implied the load and stresses within the Al film increased more sharply with increasing $w$ compared to the MLG/PVA films, and (ii) the lower ductility of the Al implies that the Al films can sustain smaller strains prior to failure. Therefore despite the Al having a much higher tensile failure stress, the Al films had a smaller load carrying capacity under Q.S. transverse loading compared to the MLG/PVA films.

\section{Loading by projectile impact}

The measured ballistic limit velocities $V_{b l}$ for the three films are plotted in Fig. 9a as a function of the projectile mass $m_{p}$. The vertical error bars indicate the two limiting velocities $V_{0}$ from which $V_{b l}$ is calculated as the mean (as described in Section 4.2). In all cases, $V_{b l}$ decreases with increasing $m_{p}$. Over the full range of $m_{p}$ values investigated here, the MLG/PVA film outperforms the equal areal mass $\mathrm{Al}$ film with a $V_{b l}$ almost twice that of the $\mathrm{Al}$ film. Moreover, the MLG/PVA film has a ballistic resistance only 20\% lower than the PVA film in spite of the PVA film having a significantly superior performance under Q.S. loading (Fig. 8a).

An image of the rear surface of the failed MLG/PVA film (see Fig. 9b.i) reveals that similar to the Q.S. case (Fig. 8b.i) the failure mode is again petalling. Moreover, an SEM micrograph (Fig. 9b.ii) shows an uneven failure surface indicative of tearing by pull-out the MLG platelets. By contrast, the failure mode of the PVA film changes from circumferential crack formation under Q.S. loading (Fig. 8b.ii) to failure by the formation of radial cracks and associated petalling (Fig. 9b.iii) under projectile impact. Further, SEM micro-graphs of the 
failure surface (Fig. 9b.iv) indicate brittle failure, revealed by the flat surface with so-called "river patterns". This embrittling of the PVA under high rate loading (also mirrored in the tensile tests, Fig. 4b) results in the failure mode changing to petalling and its ballistic limit being similar to the MLG/PVA films.

(a) Ballistic limit

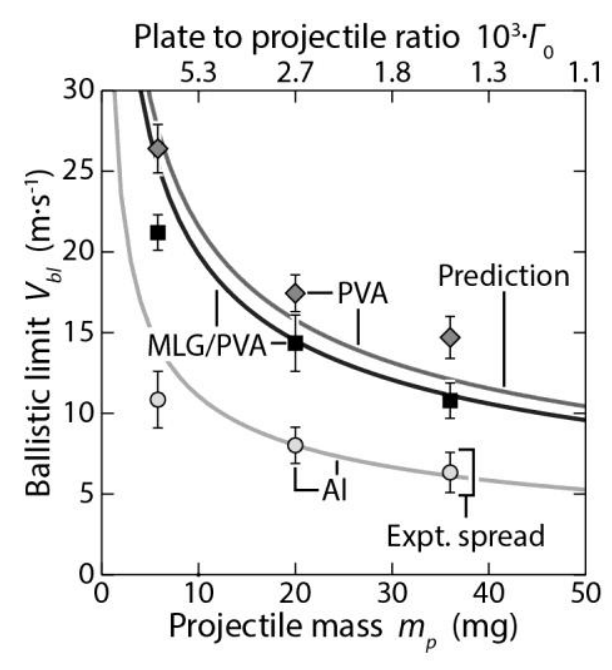

(b) Images of failed specimens
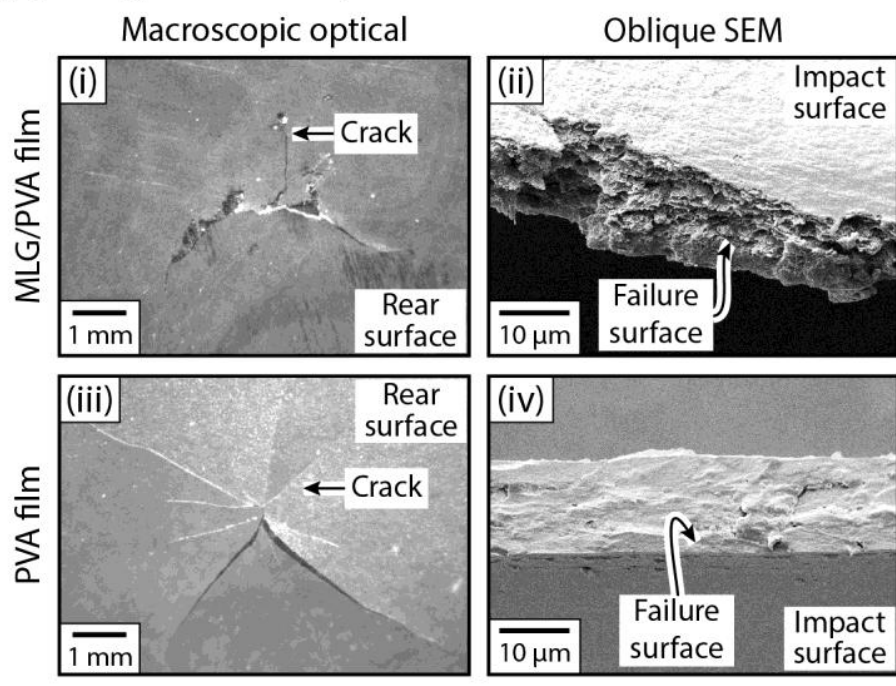

\section{(c) Rear view of MLG/PVA impact}
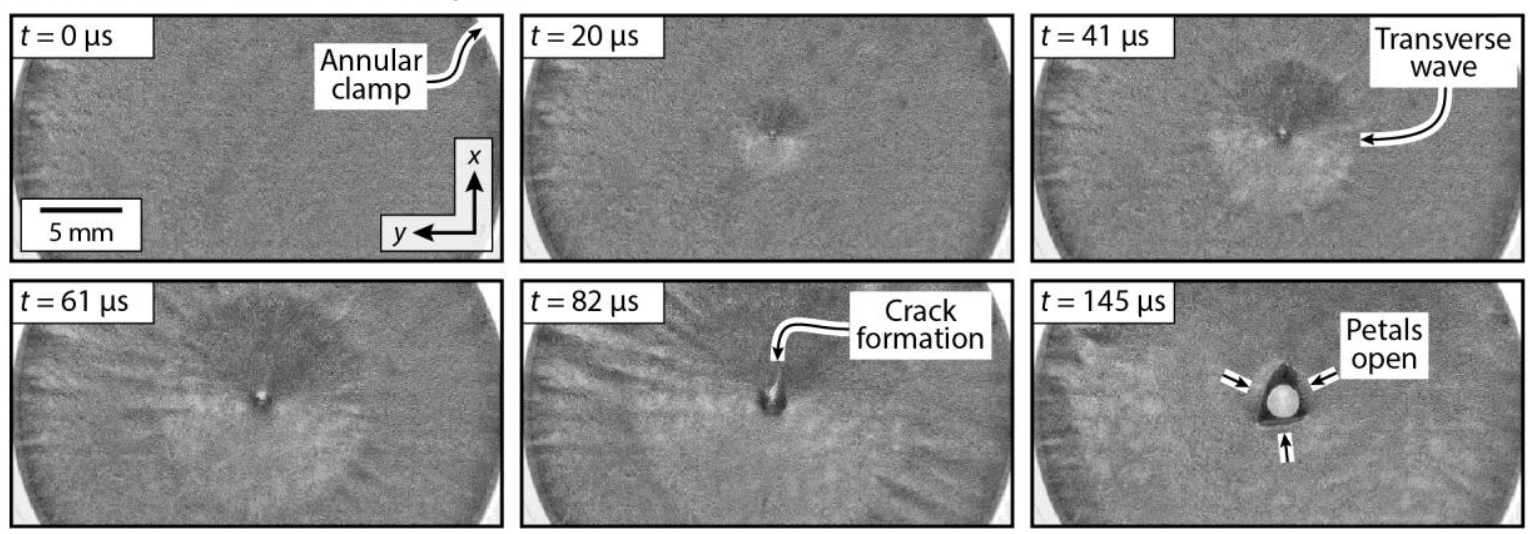

Figure 9: (a) The measured ballistic limit $V_{b l}$ (symbols) as function of the projectile mass $m_{p}$ (lower $x$-axis) or areal density ratio $\Gamma_{0}$ (upper $x$-axis). The vertical bars indicate the uncertainty in the measurement. Predictions using Eq. (3) are shown by the solid lines. (b) Images of postimpact failed MLG/PVA and PVA films (impact by a $m_{p}=20 \mathrm{mg}$ projectile at $V_{0}=16.3$ and $18.6 \mathrm{~m} \mathrm{~s}^{-1}$ for the MLG/PVA and PVA films, respectively). (c) A montage of high-speed 
photographs showing the "dead-man" view of the MLG/PVA film impacted by the $m_{p}=20 \mathrm{mg}$ projectile at $V_{0}=16.7 \mathrm{~m} \mathrm{~s}^{-1}$. Time $t=0$ is the instant the projectile impacts the film.

A high-speed photographic sequence of the rear surface (so-called "dead-man" view) of a MLG/PVA film impacted at $V_{0}=16.7 \mathrm{~m} \mathrm{~s}^{-1}$ by the $m_{p}=20 \mathrm{mg}$ projectile is shown in Fig. $9 \mathrm{c}$. A transverse elastic wave is seen to emanate from the impact site and travels towards the supports as analyzed by Phoenix and Porwal [20]. A crack is seen to initiate immediately under the projectile at $t \approx 82 \mu \mathrm{s}$, and the transverse wave impinges on the supports shortly after. The projectile is seen to come through the film by the formation of large petal cracks, $t \approx 145 \mu$ s. Very similar observations were made for the PVA and Al films, and hence high-speed images of those impact events are not shown here for the sake of brevity.

\subsection{Prediction of the ballistic resistance}

The previously discussed high-speed photography reveals that the deformation and failure modes of the films is consistent with that assumed by Phoenix and Porwal [20] in their membrane stretching analysis. Specifically, Phoenix and Porwal presented an analytical model for the ballistic limit of elastic-brittle circular membranes impacted centrally and normally by rigid projectiles. Their analysis predicted that the ballistic limit scales with the Cunniff [26] velocity $c^{*}$ via

$$
\frac{V_{b l}}{c_{x}^{*}}=2^{\frac{1}{3}} \varepsilon_{x}^{\frac{1}{12}} \frac{\left(1+\Gamma_{0}\right)}{K_{\max }^{3 / 4}},
$$

where $\varepsilon_{x} \equiv \sigma_{x} / E_{x}$ is the failure strain and the Cunniff velocity for material $x$ is given by:

$$
c_{x}^{*}=\frac{1}{2^{1 / 3}}\left(\frac{\sigma_{x}}{\rho_{x}}\right)^{2 / 3}\left(\frac{\rho_{x}}{E_{x}}\right)^{1 / 6} .
$$

Equation (3) has the strain magnification factor 


$$
K_{\max } \approx \exp \left[-\frac{4 \Gamma_{0}}{3\left(1+\Gamma_{0}\right)}\left(\psi_{\max }^{2}-1\right)\right] \psi_{\max }^{1 / 3}\left[\frac{\sqrt{\psi_{\max } / \varepsilon_{p}}\left(\psi_{\max }-1\right)}{\ln \left[1+\sqrt{\psi_{\max } / \varepsilon_{p}}\left(\psi_{\max }-1\right)\right]}\right]
$$

with the non-dimensional position $\psi_{\max } \approx \sqrt{\frac{1+\Gamma_{0}}{2 \Gamma_{0}}}$ of the transverse hinge at the instant of failure.

Predictions of $V_{b l}$ using Eq. (3) and the material parameter values for the three films as listed in Tables 1 and 2 are included in Fig. 9a. Excellent agreement is observed, confirming that the tensile membrane stretching failure mode assumed by Phoenix and Porwal [20] is the operative failure mode under projectile impact for all the three films types tested here.

\subsection{Outlook of the ballistic resistance of ultimate graphene composites}

The graphene composites manufactured and tested in this study have a performance well below the full potential due to the manufacturing issues discussed in Section 3.2. However, it is instructive to quantify the full potential of the ultimate graphene/PVA composites in anticipation of future manufacturing advances.

The strain dependence on the right-hand side of Eq. (3) is mild for practical values of failure strains $(\varepsilon \leq 0.04)$. Thus, the ballistic limit can be expressed in the form $V_{b l} / c_{x}^{*}=f\left(\Gamma_{0}\right)$ as first hypothesized by Cunniff [26]; i.e. the non-dimensional ballistic limit $V_{b l} / c_{x}^{*}$ is only a function of the areal mass ratio $\Gamma_{0}$, and therefore the material metric $c_{x}^{*}$ characterizes the ballistic resistance capacity of a material $x$ when membrane stretching is the operative failure mechanism. The experiments reported here suggest membrane stretching is expected to be the operative failure mode of graphene composites, and therefore we shall use this model to quantify the ballistic potential of ultimate graphene/PVA composites.

Equation (2) can be employed to predict the in-plane modulus $E_{c}$ and strength $\sigma_{c}$ of the ultimate graphene/PVA composites discussed in Section 3.2. Using Eq. (3), Fig. 10a plots their predicted Cunniff velocity $c_{c}^{*}$ as a function of graphene volume fraction $\phi_{g}$ (constituent densities 
are given in Table 1 and mechanical properties in Table 2). The Cunniff velocity increases with increasing $\phi_{g}$ and is remarkably high even at relatively small volume fractions of graphene.

(a) Effect of graphene vol. fraction

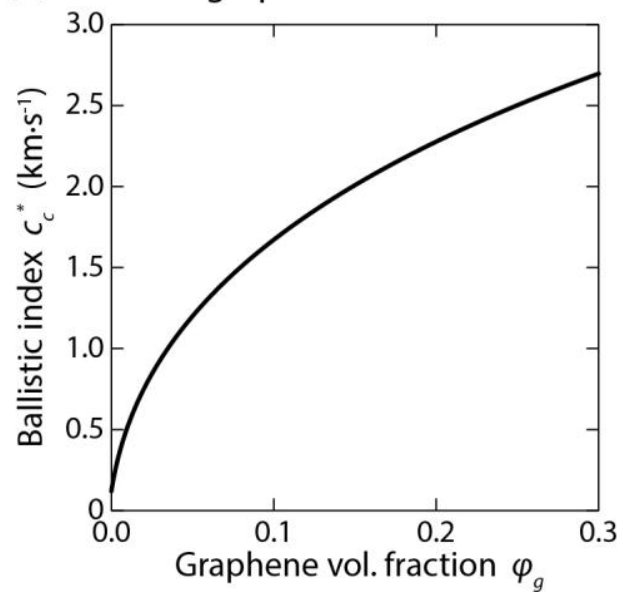

(b) Material comparison

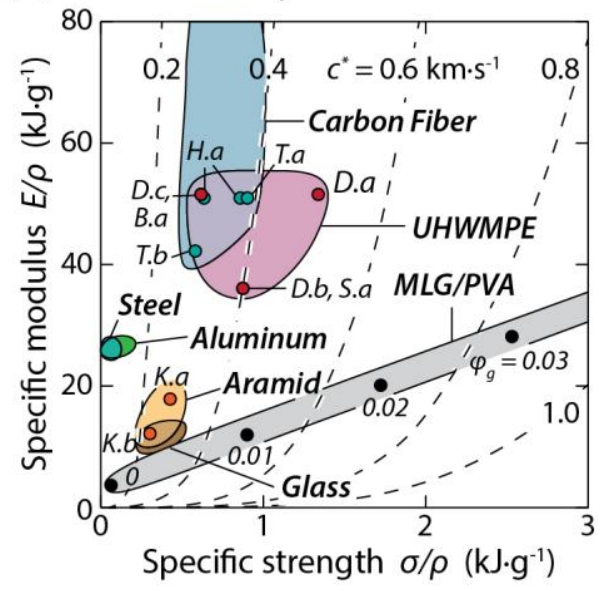

Figure 10: (a) Prediction of the Cunniff velocity $c_{c}^{*}$ versus graphene volume fraction $\phi_{g}$ for ultimate graphene/PVA composites. (b) A plot with axes of specific strength and stiffness and contours of $c^{*}$. Included are high performance commercial composites and ultimate graphene/PVA composites with $\phi_{g} \leq 3.5 \%$. The abbreviations and references in (b) are listed in the footnote ${ }^{1}$.

In order to illustrate the remarkable performance of these ultimate graphene composites, Fig. 10b plots an Ashby chart with axes of (density) specific strength and modulus. Contours of $c^{*}$ are marked on the chart along with the locations of key high-performance commercial

1 Aluminum (2024 and 7075 [22]); Aramid: K.a (Gold Shield GV2018; tested following procedure in [28]), K.b (Kevlar $290^{\circ} / 90^{\circ}$ laminate [29]); Carbon fiber: H.a (Hexply 8552 [18,30]), T.a (Toray T800H $0^{\circ} / 90^{\circ}$ laminate [31]), T.b (Toray T300 0 $/ 90^{\circ}$ laminate [32]); Glass (E and S2 glass $0^{\circ} / 90^{\circ}$ laminate [22]); Steel (AISI 1020 and AISI 4340 [22]); and UHWMPE: B.a (Tensylon HSBD30A [28]), D.a (Dyneema HB212 [28]), D.b (Dyneema HB50 [33]), D.c (Dyneema BT10 [33]), S.a (Spectra Shield II SR3136 [34]). 
composites currently used for ballistic resistance. These include aramid, carbon, glass and polyethylene (UHMWPE) fiber based composites, in addition to some widely used metals such as steel and aluminum. The predicted locations of graphene/PVA composites, with $0 \leq \phi_{g} \leq$ $3.5 \%$, is also marked. This indicates the remarkable potential of graphene/PVA composites as ballistic materials: the best current materials have a $c^{*} \leq 500 \mathrm{~m} \mathrm{~s}^{-1}$, while Fig. 10b suggests that even an addition of 1 vol.\% graphene into PVA is capable of surpassing this value. In fact, a $\phi_{g}=0.3$ graphene/PVA composite is predicted to have a $c^{*}$ five times the best current composites. Graphene composites thus have an enormous potential as ballistic resistant materials; though it remains to be seen whether such defect-free composites with large and aligned flake are manufacturable on a scales to make them practically viable.

\section{Concluding remarks}

A method to manufacture composites comprising a polyvinyl alcohol (PVA) matrix reinforced by $\sim 35$ vol.\% multilayer graphene (MLG) was developed. The MLG comprised $\sim 30$ graphene layers and the resulting conductive composite had a reduced strain-rate sensitivity and higher strengths at lower strain rates compared to the PVA matrix. The ductility of the composite, however, was lower than the PVA, although the toughness of the composite was similar to that of aluminum. Nevertheless, the strength and stiffness of the composite are well below the predicted theoretical upper bounds. This is presumably due to defects such as poor platelet alignment and small graphene platelet sizes.

Edge clamped films of the composite had a ballistic limit that was nearly twice that of equal areal mass aluminum foils over the whole range of projectile masses investigated here. This ballistic limit is shown to be well captured by a membrane stretching analysis. This analysis is used to predict the ballistic performance of so-called "ultimate" graphene composites 
comprising large aligned flakes that attain the theoretical maximum mechanical properties. Such composites, if manufacturable, have the potential to have a ballistic limit in excess of three times the best high-performance commercial composites with only 10 vol.\% graphene reinforcement in PVA.

\section{Acknowledgements}

The work was supported by the Office of Naval Research Grant N62909-15-1-N058 (Program manager, Dr. Judah Goldwasser). Dr B.P. Russell was supported by a Ministry of Defence/Royal Academy of Engineering Research Fellowship (Grant Number RG60007). The authors also wish to acknowledge the Mr. S. Marshall in the fabrication of the pressure filtration and two-stage miniature gas gun devices used in this study.

\section{Appendix A: Conductivity measurement}

The electrical conductivity $\Sigma_{c}$ of the MLG/PVA films was measured using four-terminal sensing (Fig. A1). The current $I$ supplied at the outer two connections was varied from 5 to 50 $\mu \mathrm{A}$ and was measured to a $0.1 \mu \mathrm{A}$ precision using an ammeter placed in series with the current source. The voltage $V$ was sensed across the inner connections using a voltmeter with a sensitivity of $0.01 \mathrm{~V}$. The voltage was observed to vary linearly with the imposed current, and hence the $\Sigma_{c}$ was calculated via

$$
\Sigma_{c}=\frac{I}{V} \frac{L}{W t_{c}},
$$

where $L=20 \mathrm{~mm}$ is the separation distance between the inner sensing probes, $W=10 \mathrm{~mm}$ is the width of the film and $t_{c}$ is the effective thickness of the film given in Table 2. 


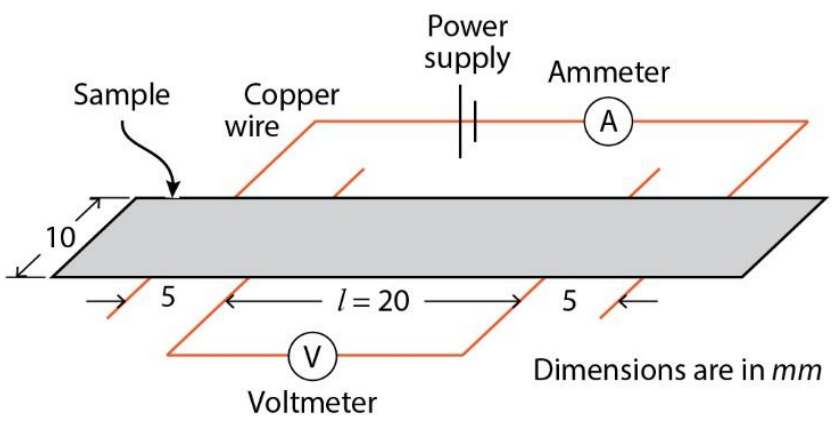

Figure A1: Sketch of the four-terminal sensing experiment used to measure the conductivity of the MLG/PVA composite films.

\section{References}

[1] M. Xu, T. Liang, M. Shi, H. Chen, Graphene-Like Two-Dimensional Materials, Chem. Rev. 113 (2013) 3766-3798. doi:10.1021/cr300263a.

[2] K.S. Novoselov, V.I. Fal'ko, L. Colombo, P.R. Gellert, M.G. Schwab, K. Kim, A roadmap for graphene, Nature. 490 (2012) 192-200. doi:10.1038/nature11458.

[3] M. Yi, Z. Shen, A review on mechanical exfoliation for the scalable production of graphene, J. Mater. Chem. A. 3 (2015) 11700-11715. doi:10.1039/C5TA00252D.

[4] K.R. Paton, E. Varrla, C. Backes, R.J. Smith, U. Khan, A. O’Neill, C. Boland, M. Lotya, O.M. Istrate, P. King, T. Higgins, S. Barwich, P. May, P. Puczkarski, I. Ahmed, M. Moebius, H. Pettersson, E. Long, J. Coelho, S.E. O’Brien, E.K. McGuire, B.M. Sanchez, G.S. Duesberg, N. McEvoy, T.J. Pennycook, C. Downing, A. Crossley, V. Nicolosi, J.N. Coleman, Scalable production of large quantities of defect-free few-layer graphene by shear exfoliation in liquids, Nat Mater. 13 (2014) 624-630. doi:10.1038/nmat3944.

[5] H. Kim, A.A. Abdala, C.W. Macosko, Graphene/Polymer Nanocomposites, Macromolecules. 43 (2010) 6515-6530. doi:10.1021/ma100572e.

[6] J.R. Potts, D.R. Dreyer, C.W. Bielawski, R.S. Ruoff, Graphene-based polymer nanocomposites, Polymer. 52 (2011) 5-25. doi:10.1016/j.polymer.2010.11.042.

[7] C.N.R. Rao, A.K. Sood, K.S. Subrahmanyam, A. Govindaraj, Graphene: The New TwoDimensional Nanomaterial, Angewandte Chemie International Edition. 48 (2009) 77527777. doi:10.1002/anie.200901678.

[8] V. Singh, D. Joung, L. Zhai, S. Das, S.I. Khondaker, S. Seal, Graphene based materials: Past, present and future, Progress in Materials Science. 56 (2011) 1178-1271. doi:10.1016/j.pmatsci.2011.03.003.

[9] J. Liang, Y. Huang, L. Zhang, Y. Wang, Y. Ma, T. Guo, Y. Chen, Molecular-Level Dispersion of Graphene into Poly(vinyl alcohol) and Effective Reinforcement of their Nanocomposites, Adv. Funct. Mater. 19 (2009) 2297-2302. doi:10.1002/adfm.200801776.

[10] X. Zhao, Q. Zhang, D. Chen, Enhanced Mechanical Properties of Graphene-Based Poly(vinyl alcohol) Composites, Macromolecules. $43 \quad$ (2010) 2357-2363. doi:10.1021/ma902862u. 
[11] X. Yang, L. Li, S. Shang, X. Tao, Synthesis and characterization of layer-aligned poly(vinyl alcohol)/graphene nanocomposites, Polymer. 51 (2010) 3431-3435. doi:10.1016/j.polymer.2010.05.034.

[12] J. Wang, X. Wang, C. Xu, M. Zhang, X. Shang, Preparation of graphene/poly(vinyl alcohol) nanocomposites with enhanced mechanical properties and water resistance, Polym. Int. 60 (2011) 816-822. doi:10.1002/pi.3025.

[13] S. Morimune, T. Nishino, T. Goto, Poly(vinyl alcohol)/graphene oxide nanocomposites prepared by a simple eco-process, Polym J. 44 (2012) 1056-1063. doi:10.1038/pj.2012.58.

[14] J.-H. Lee, P.E. Loya, J. Lou, E.L. Thomas, Dynamic mechanical behavior of multilayer graphene via supersonic projectile penetration, Science. 346 (2014) 1092-1096. doi:10.1126/science. 1258544 .

[15] M.E. Backman, W. Goldsmith, The mechanics of penetration of projectiles into targets, International Journal of Engineering Science. 16 (1978) 1-99. doi:10.1016/00207225(78)90002-2.

[16] R. Vermorel, N. Vandenberghe, E. Villermaux, Impacts on thin elastic sheets, Proceedings of the Royal Society of London A: Mathematical, Physical and Engineering Sciences. 465 (2009) 823-842. doi:10.1098/rspa.2008.0297.

[17] B.A. Cheeseman, T.A. Bogetti, Ballistic impact into fabric and compliant composite laminates, Composite Structures. 61 (2003) 161-173. doi:10.1016/S0263-8223(03)00029-1.

[18] K. Karthikeyan, B.P. Russell, N.A. Fleck, M.R. O’Masta, H.N.G. Wadley, V.S. Deshpande, The soft impact response of composite laminate beams, International Journal of Impact Engineering. 60 (2013) 24-36. doi:10.1016/j.ijimpeng.2013.04.002.

[19] K. Karthikeyan, B.P. Russell, Polyethylene ballistic laminates: Failure mechanics and interface effect, Mater Des. 63 (2014) 115-125. doi:10.1016/j.matdes.2014.05.069.

[20] S.L. Phoenix, P.K. Porwal, A new membrane model for the ballistic impact response and V50 performance of multi-ply fibrous systems, International Journal of Solids and Structures. 40 (2003) 6723-6765. doi:10.1016/S0020-7683(03)00329-9.

[21] C. Lee, X. Wei, J.W. Kysar, J. Hone, Measurement of the Elastic Properties and Intrinsic Strength of Monolayer Graphene, Science. 321 (2008) 385-388. doi:10.1126/science.1157996.

[22] CES EduPack, Granta Design Limited, 2015.

[23] B.D. Cullity, S.R. Stock, Elements of X-Ray Diffraction, Third Edition, Prentice Hall, Upper Saddle River, NJ, 2001.

[24] V. Palermo, I.A. Kinloch, S. Ligi, N.M. Pugno, Nanoscale Mechanics of Graphene and Graphene Oxide in Composites: A Scientific and Technological Perspective, Adv. Mater. (2016) n/a-n/a. doi:10.1002/adma.201505469.

[25] ASTM D1938, Standard Test Method for Tear-Propagation Resistance (Trouser Tear) of Plastic Film and Thin Sheeting by a Single-Tear Method, ASTM International, West Conshohocken, PA, 2014. www.astm.org.

[26] P.M. Cunniff, Dimensionless parameters for optimization of textile-based body armor systems, in: W.G. Reinecke (Ed.), Proceedings of the 18th International Symposium on Ballistics, Technomic Publishing Company, Inc, San Antonio, TX, 1999: pp. 1303-1310.

[27] R. Vermorel, N. Vandenberghe, E. Villermaux, Radial Cracks in Perforated Thin Sheets, Phys. Rev. Lett. 104 (2010) 175502. doi:10.1103/PhysRevLett.104.175502. 
[28] M.R. O’Masta, D.H. Crayton, V.S. Deshpande, H.N.G. Wadley, Indentation of polyethylene laminates by a flat-bottomed cylindrical punch, Composites Part A: Applied Science and Manufacturing. 80 (2016) 138-147. doi:10.1016/j.compositesa.2015.10.015.

[29] A.K. Bandaru, V.V. Chavan, S. Ahmad, R. Alagirusamy, N. Bhatnagar, Ballistic impact response of Kevlar® reinforced thermoplastic composite armors, International Journal of Impact Engineering. 89 (2016) 1-13. doi:10.1016/j.ijimpeng.2015.10.014.

[30] Hexcel Corp., HexPly 8552: Product Data, (2016). http://www.hexcel.com/Resources/DataSheets/Prepreg-Data-Sheets/8552_eu.pdf (accessed August 15, 2016).

[31] Toray Carbon Fibers America, Inc., T800H Data Sheet, 2016. http://www.toraycfa.com/pdfs/T800HDataSheet.pdf (accessed August 16, 2016).

[32] Toray Carbon Fibers America, Inc., T300 Data Sheet, 2016. http://www.toraycfa.com/pdfs/T300DataSheet.pdf (accessed August 16, 2016).

[33] M.R. O’Masta, D.H. Crayton, V.S. Deshpande, H.N.G. Wadley, Mechanisms of penetration in polyethylene reinforced cross-ply laminates, International Journal of Impact Engineering. 86 (2015) 249-264. doi:10.1016/j.ijimpeng.2015.08.012.

[34] M.R. O’Masta, V.S. Deshpande, H.N.G. Wadley, Defect controlled transverse compressive strength of polyethylene fiber laminates, International Journal of Solids and Structures. 52 (2015) 130-149. doi:10.1016/j.ijsolstr.2014.09.023. 
Multi-layer graphene / Polyvinyl alcholol (MLG/PVA) composite

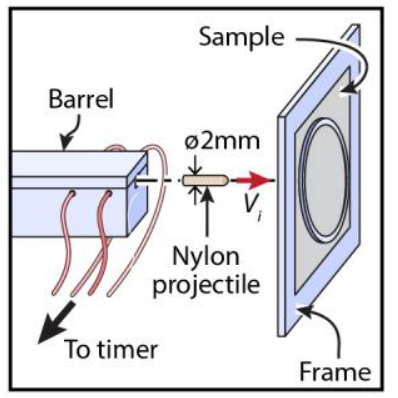

$c$ : Ballistic resistance index

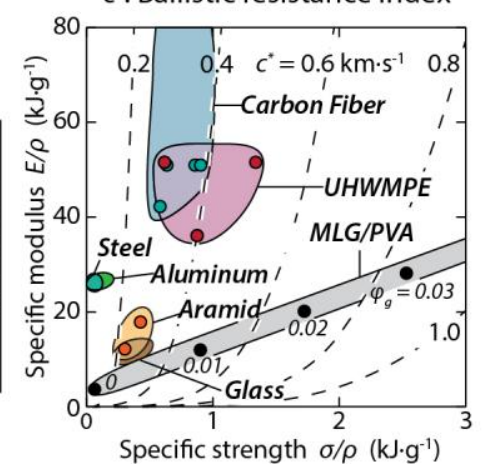

